Introduction: Mucinous ovarian carcinomas are less common than serous and endometriod type, and are more frequently confined to the ovary at the time of diagnosis. But primary signet ring cell mucinous carcinomas of the ovary are extremely rare.

Case Presentation: A 40 yr old patient presented with extremely rare primary signet cell mucinous carcinoma of ovary. She presented with abdominal distension and frequency of urination for one month. She was evaluated and CECT whole abdomen was s/o large left ovarian mass. All the tumor markers were with in normal range. Laparotomy frozen section of left adnexal mass was done and was reported as malignant with sheets of signet ring cells seen. Hence complete staging laparotomy including TAH with RSO with bilateral pelvic lymph node dissection with total omentectomy with para aortic lymph node dissection. Final histopathology with IHC markers were $S / O$ primary signet ring cell carcinoma of ovary with no extracapsular invasion, no lymph nodal involvement \& no metastatic spread.

Conclusion: We present a very rare case of primary signet ring cell of ovary, confined to ovary itself. On literature review only 14 cases have been reported and of them very few are malignant.

\section{Missed Abstracts}

\section{Clinical presentation and management of malignant germ cell ovarian tumours in BPKMCH \\ Jitendra Pariyar, Binuma Shrestha ${ }^{1}$ \\ Department of Gynecologic Oncology, Civil Service Hospital, Kathmandu, ${ }^{1}$ Gynecologic Oncology Unit, B.P. Koirala Memorial Cancer Hospital, Bharatpur, Chitwan, Nepal}

Background: Germ cell malignancies account for about $5 \%$ of all ovarian cancers. These tumours grow rapidly and often produce symptoms quicker than the slow growing epithelial tumour. Commonly seen in the first two decades of life germ cell malignancies are highly chemosensitive and are potentially curable with surgery and chemotherapy. This study is the first of its kind regarding the epidemiology, management and outcome of patients with malignant germ cell tumour in Nepal.

Objective: To analyze the clinical presentation and management outcomes of malignant germ cell tumours managed in B.P. Koirala Memorial Cancer Hospital, Nepal.

Methodology: Descriptive study conducted in B.P. Koirala Memorial Cancer Hospital, Nepal. Case records of malignant germ cell tumours attending the hospital from January 1999 to December 2009 were analyzed regarding their illness history, clinical examination, investigations, treatment, follow-up and outcomes measured.

Observations: Total 65 cases of malignant germ cell tumours with age range from 2 to 58 years (mean 21.7 years) were received. $42 \%$ cases were TibetoBurmese; $30 \%$ were Indo-Aryans. There were 15 cases (23\%) of dysgeminoma, 21 endodermal sinus tumor (32\%), 16 Immature Cystic Teratoma (24.5\%), 9 (14\%) Mixed Germ Cell, 2 unclassified GCT (3.5\%) and 2 malignant transformation in teratoma (3.5\%). 33 (49.5\%) patients had early stage disease, 37 (57\%) underwent fertility preserving surgery. 4 cases (9\%) due to disseminated disease, underwent neoadjuvant chemotherapy followed by debulking surgery. 51 cases (78.5\%) received adjuvant chemotherapy (BEP or EP regimen). The overall survival was $70 \%$.

Conclusion: Early stage germ cell malignancies can be safely managed by fertility preserving surgery followed by, chemotherapy if indicated. For advanced diseases, neoadjuvant chemotherapy followed by surgery can be undertaken with curable intent.

\section{Cervix: Poster Abstract}

Clinicopathological correlates and need for adjuvant radiotherapy in early stage carcinoma cervix

Apoorva Reddy, Shalini Rajaram, Bindiya Gupta, Shipra Garg' ${ }^{1}$ Kiran Mishra ${ }^{1}$, Neerja Goel, Gita Radhakrishnan, N. B. Vaid Departments of Obstetrics and Gynaecology and ${ }^{1}$ Pathology, Guru Tegh Bahadur Hospital, UCMS, New Delhi, India
Objective: To analyse clinical-pathological findings in women undergoing surgery for early stage carcinoma cervix and to determine the need for adjuvant radiotherapy.

Methods: The study was a retrospective one done to analyse data of women who had been operated for carcinoma cervix in the last 10 years at a tertiary hospital in Delhi. Type II/ III radical hysterectomy with pelvic lymphadenectomy was done depending on stage. Histopathological findings and need for adjuvant therapy based on presence of clinical and pathological criteria were studied.

Results: A total of 93 eligible patient records were included in the study. Mean age and parity was $48.09 \pm 22.36$ years and $4.29 \pm 3.9$ respectively. $47.31 \%$ (44) of the women had stage 1B1 carcinoma cervix; 31.18\% (29) had stage 1B2 and $19.35 \%$ (18) had stage $2 \mathrm{~A}$ disease. Average size of the tumour growth was $3.25 \pm 2.46 \mathrm{~cm}$ with the largest being $7 \mathrm{~cm}$. Keratinising squamous cell carcinoma was seen in $48.38 \%$ (45) of patients whereas the non-keratinising one was seen in $33.33 \%$ (31). Large cell variant was seen in $6.45 \%$ (6) whereas micro invasive cancer was seen in 4 women. Adenocarcinoma constituted only $3.2 \%$ (3) of the study group. Vaginal cuff involvement was present in $9.67 \%$ (9) of patients and an equal number had occult parametrial invasion. A significant number of these had stage 2A disease (16.66\% and $22.22 \%$ respectively). Between $1 \mathrm{~B} 1$ and $1 \mathrm{~B} 2$ there was no significant difference in the incidence of vaginal cuff involvement and occult parametrial invasion ( $P$ equals to 0.206 and 1 respectively). Lymph nodes were positive in $24.73 \%$ with the obturator lymph node being the commonest involved (17). In stage 1b1 25\% (11) had positive lymph nodes; in stage 1B2 34.48\% (10) and in 2A only one woman had positive pelvic lymph nodes. This difference was also not significant $(\mathrm{P}=0.434)$. About 50\% (47) of patients were referred for adjuvant radiotherapy on the basis of the pathological findings. 61\% (11) of patients with stage $2 \mathrm{~A}$ disease, $51.7 \%$ (15) of patients with stage $1 \mathrm{~b} 2$ and $45.45 \%$ (20) with stage $1 \mathrm{~B} 1$ disease required adjuvant radiotherapy.

Conclusion: There was no significant difference in the pathological findings in patients of $1 \mathrm{~B} 1$ and $1 \mathrm{~B} 2$. Also the need for radiotherapy in both the groups was similar. Hence similar surgical approach to women with both $1 \mathrm{~B} 1$ and 1B2 disease appears appropriate.

\section{Cervix: Poster Abstract}

Cervical cancer management in Rural India: Are we really living in $21^{\text {st }}$ century or need to focus on health education of our doctors

\section{Sujata Mittal}

\section{Paras Hospital, Gurgaon, Haryana, India}

Objectives: To study cases of cervical cancer managed/unmanaged in rural India and to analyze the reasons for poor outcome.

Methods: This is a retrospective study of 218 cases of cervical cancers between 2008-2013 with resultant outcome in terms of treatment or absence of treatment in spite of diagnosis. Reasons for not taking the treatment have been analyzed. Also, analysis of 21 cases of simple hysterectomy with resultant complications like WVF, RVF has been done. Indications of surgery, operating surgeon, availability of preoperative/postoperative HPR, slides/ blocks, discharge summary and disease status at the time of referral was done. Results: $44 \%$ refused to take treatment in spite of stage III diagnosis citing financial constraints, distance to be traveled daily for RT and apathetic attitude of family towards females. $20.65 \%$ opted for other hospitals. $29.8 \%$ took complete treatment. $80 \%$ of females were illiterate and dependent. $9.7 \%$ had simple hysterectomy for invasive disease. 95\% of simple hysterectomies were performed by general surgeons in private setups resulting in $19 \%$ of complications like VVF, RVF. $100 \%$ cases of simple Hysterectomy did not have pre-operative biopsy. Only $50 \%$ cases had post-operative biopsy report and in none of the cases were slide/blocks available for review as trained pathologists were not available. General surgeons who had performed surgery were neither trained in doing P/V examinations nor aware of staging of cervical cancer. Conclusion: Illiteracy, poverty and absence of implementation of cancer control programs are the major hurdles in control of cervical cancer. The study highlights the absence of Government's will to control cervical cancer in rural India. It emphasizes on the need of intensive training and health education of gynaecologists and surgeons at district/rural level, lack of which is a primary factor for violation of medical ethics by the doctors. 